\title{
Photoinduced phase separation and miscibility in the condensed phase of a mixed Langmuir monolayer
}

\author{
P. Viswanath and K. A. Suresh* \\ Raman Research Institute, Sadashivanagar, Bangalore - 560080.
}

\section{Supporting Information}

*Corresponding author. E-mail: suresh@rri.res.in 


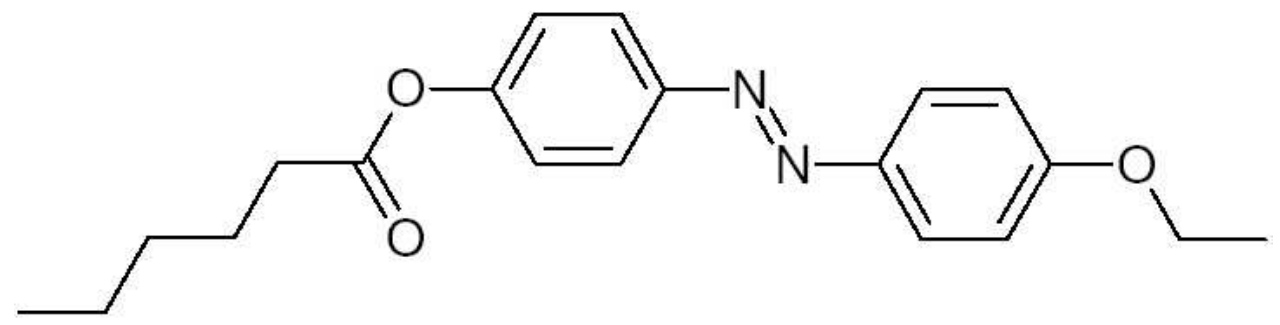

(a)

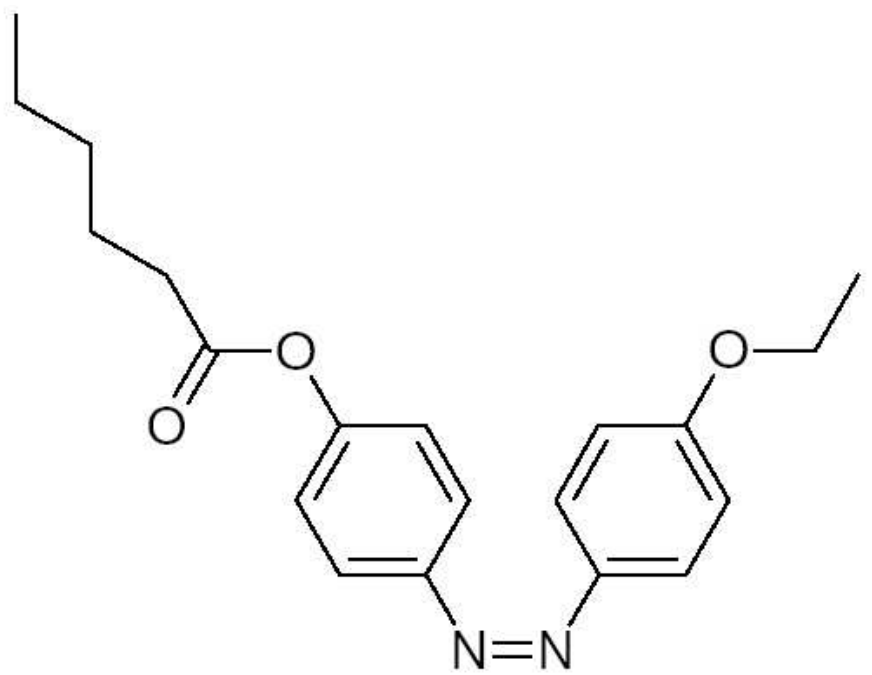

(b)

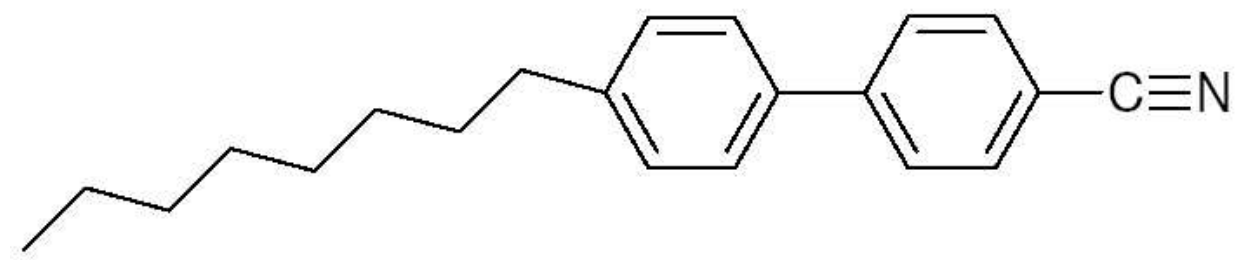

(c)

Figure S 1: Structures of EPPH isomers and 8CB. Figure (a) shows the EPPH isomer in the trans state. Figure (b) shows the EPPH isomer in the cis state. Figure (c) shows the 8CB molecule. 


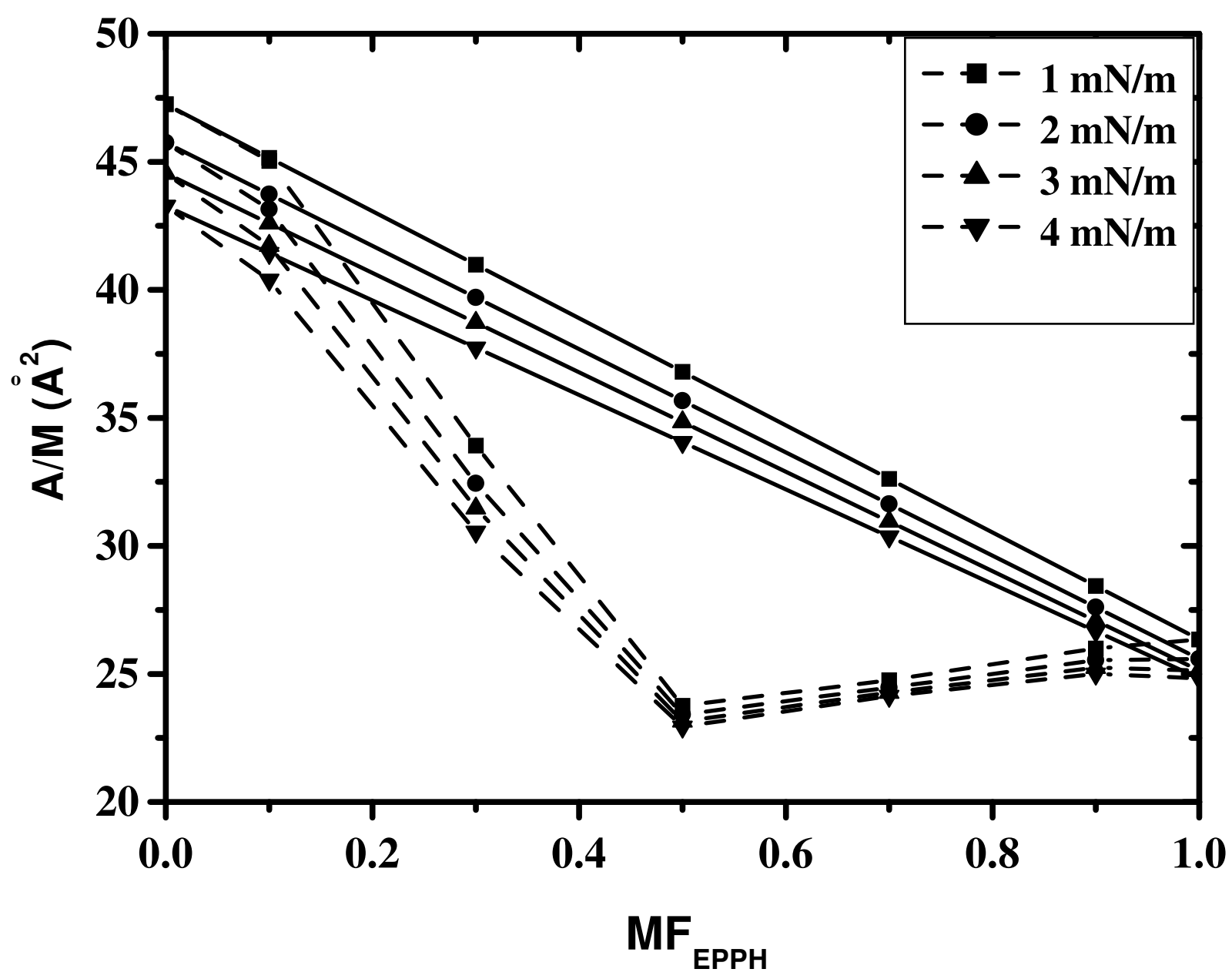

Figure S 2: Variation of experimentally obtained $\mathrm{A} / \mathrm{M}, \mathrm{A}_{12}$ (dashed lines) and computed ideal $\mathrm{A} / \mathrm{M}, \mathrm{A}_{i d}$ (continuous lines) with mole fraction of EPPH in $8 \mathrm{CB}$ for different surface pressures. The excess area, $\mathrm{A}_{e x c}=A_{12}-A_{i d}$, gives a value of about $-13 \AA^{2}$ at $0.5 \mathrm{MF}$ of $\mathrm{EPPH}$ in $8 \mathrm{CB}$. 


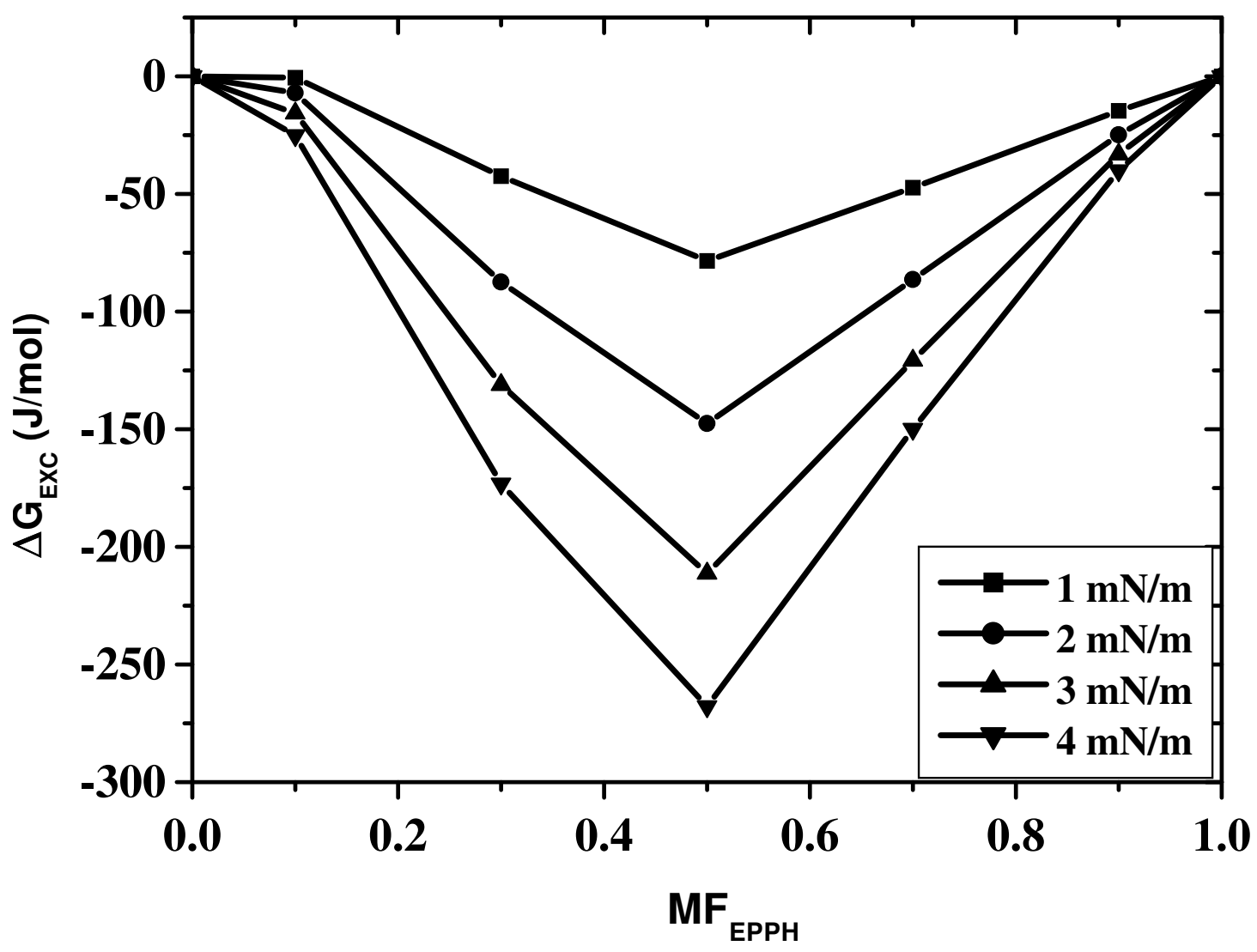

Figure S 3: Variation of the excess Gibbs free energy, $\Delta G_{E X C}$ with mole fraction (MF) of EPPH in $8 \mathrm{CB}$ at different surface pressures. At $0.5 \mathrm{MF}$ of $\mathrm{EPPH}$ in $8 \mathrm{CB}$, a pronounced minimum is seen for different surface pressures. It has a value of about $-275 \mathrm{~J} / \mathrm{mol}$ at a surface pressure of $4 \mathrm{mN} / \mathrm{m}$.

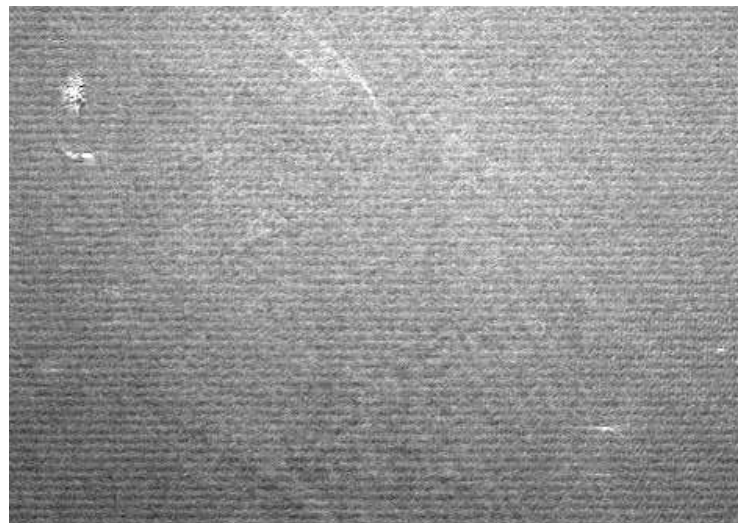

Figure S 4: BAM image for $0.5 \mathrm{MF}$ of $\mathrm{EPPH}$ in $8 \mathrm{CB}$ showing a homogeneous and uniformly covered monolayer at an $A / M$ of $22.1 \AA^{2}$. The bright streaks and the spots are artifacts due to the black substrate placed in the subphase to prevent reflection. Scale of the image is $4.8 \mathrm{x}$ $3.2 \mathrm{~mm}^{2}$. 


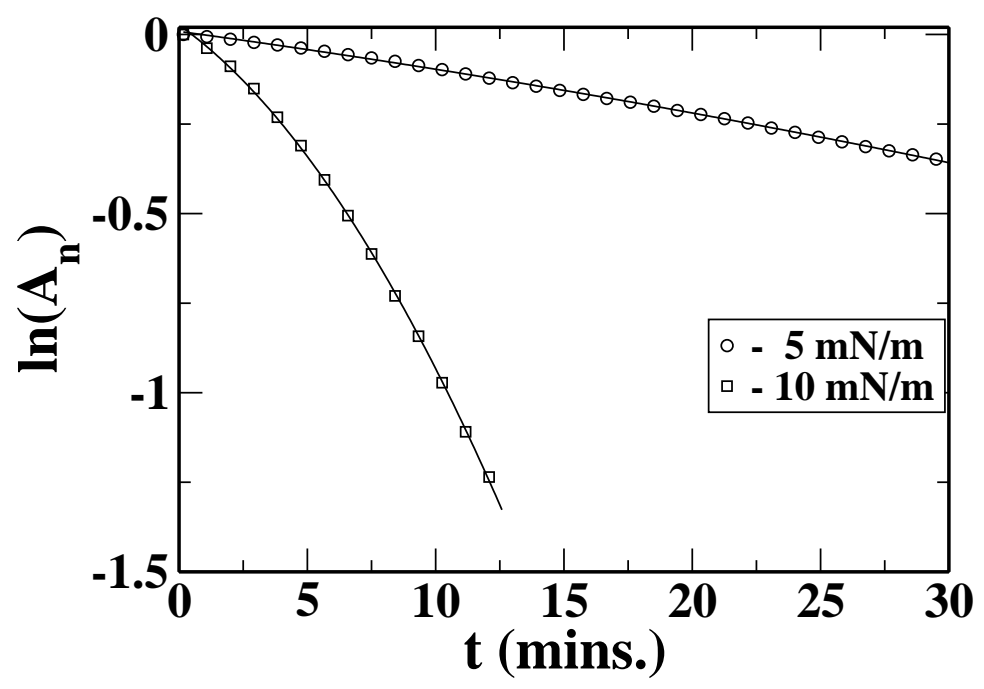

Figure S 5: Variation of the logarithm of the normalized area $\left(\mathrm{A}_{n}\right)$ with time $(t)$ at a given surface pressure for EPPH monolayer. The symbols are the experimental data points and the continuous lines are the fits for the equation, $\ln \left(A_{n}\right)=-\alpha * t-\beta * t^{2}$. The fit coefficients $\alpha, \beta$ and the correlation coefficient $(r)$ for the surface pressures, $5 \mathrm{mN} / \mathrm{m}$ and $10 \mathrm{mN} / \mathrm{m}$ are, $\alpha=-0.0086, \beta=-0.0001, r=0.9996$ and $\alpha=-0.0426, \beta=-0.0051, r=0.9997$ respectively.

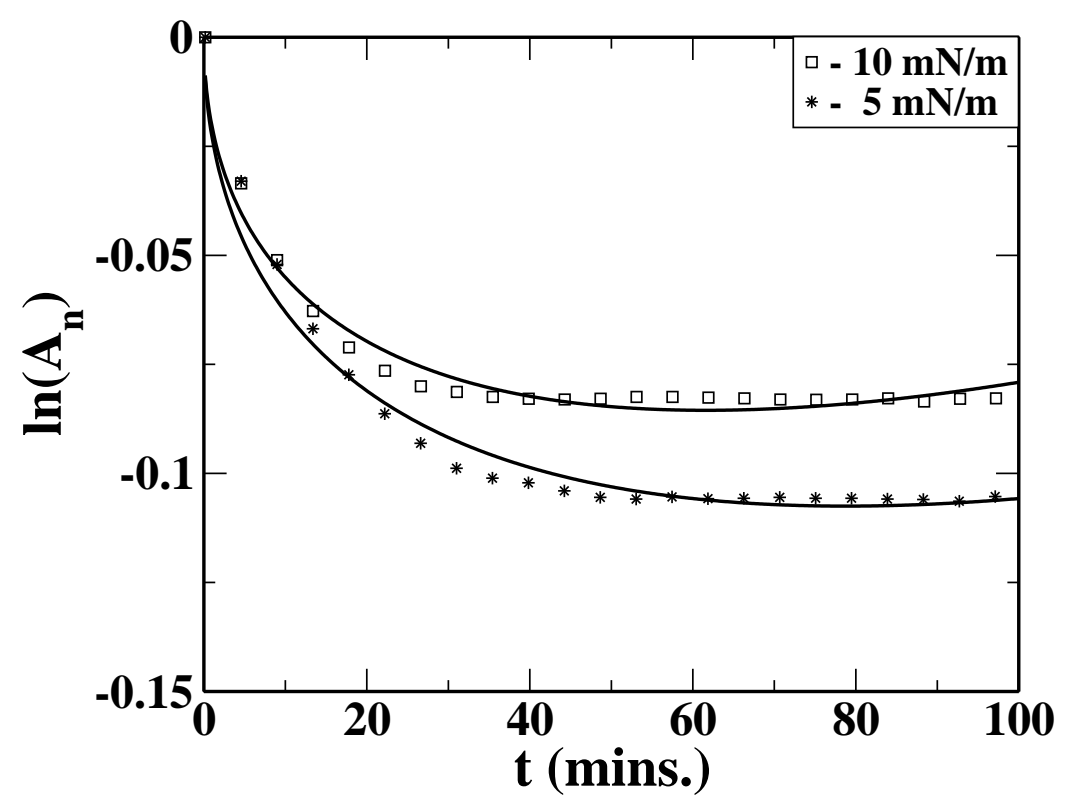

Figure S 6: Variation of the logarithm of the normalized area $\left(\mathrm{A}_{n}\right)$ with time $(t)$ at a given surface pressure for $0.5 \mathrm{MF}$ of $\mathrm{EPPH}$ in $8 \mathrm{CB}$. The symbols are the experimental data points and the continuous lines are the fits for the equation, $\ln \left(A_{n}\right)=a * t^{0.5}+b * t$. The fit coefficients $a, b$ and the correlation coefficient $(r)$ for the surface pressures, $10 \mathrm{mN} / \mathrm{m}$ and $5 \mathrm{mN} / \mathrm{m}$ are $a=-0.0218, b=0.00139, r=0.981$ and $a=-0.0242, b=0.00137, r=0.989$ respectively. 


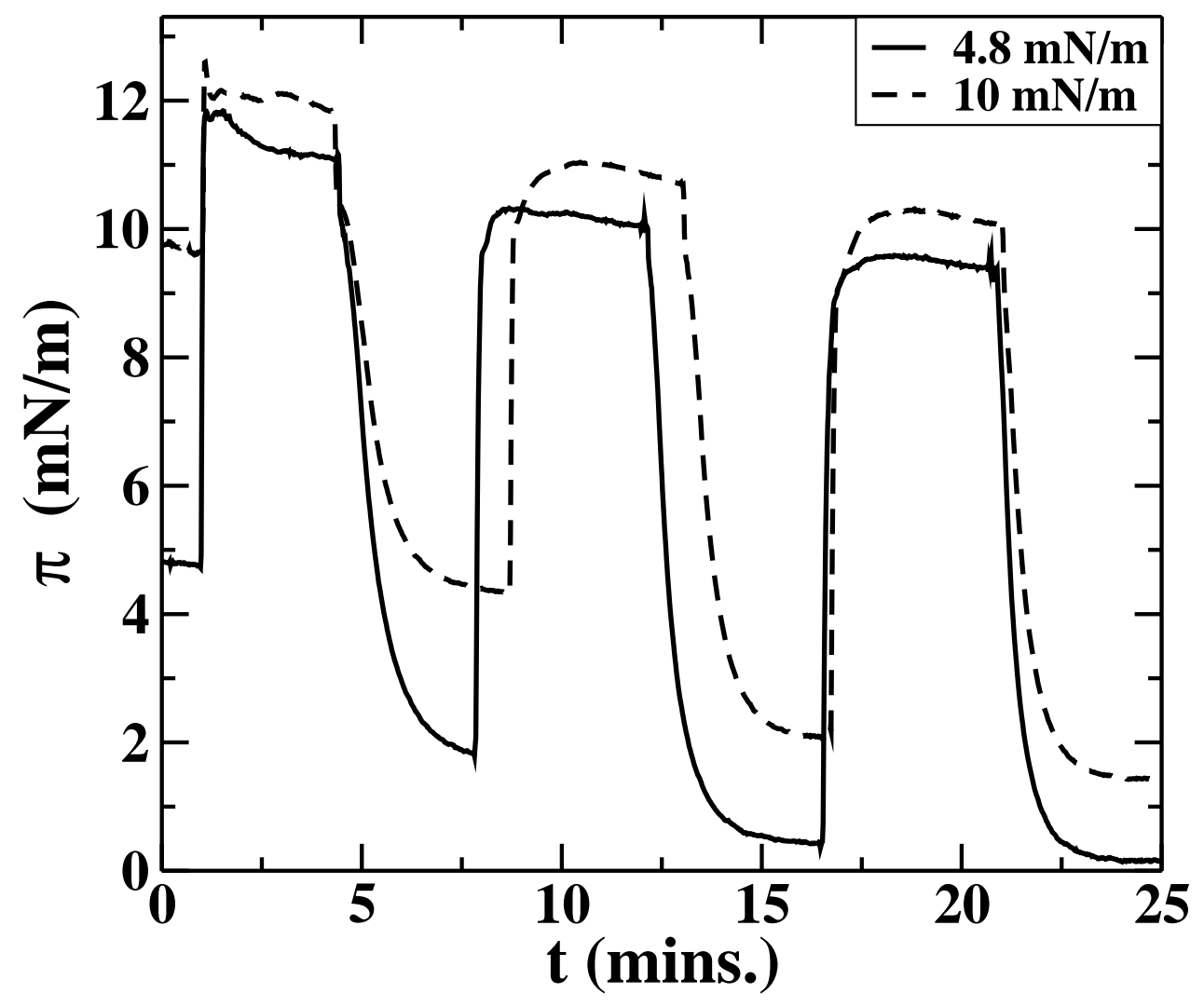

Figure S 7: Variation of the surface pressure $(\pi)$ with time $(t)$ for $0.7 \mathrm{MF}$ of EPPH in $8 \mathrm{CB}$ on alternately illuminating with UV and visible radiations. Here for the initial surface pressure of $4.8 \mathrm{mN} / \mathrm{m}$, the peak value in surface pressure of about $11 \mathrm{mN} / \mathrm{m}$ decreases to about $9.4 \mathrm{mN} / \mathrm{m}$ for three cycles of illumination. The dip from the initial surface pressure, decreases to about $0 \mathrm{mN} / \mathrm{m}$ for three cycles of illumination. Similar decreasing trends are seen in the peak and the dip values for an initial surface pressure of $10 \mathrm{mN} / \mathrm{m}$. 